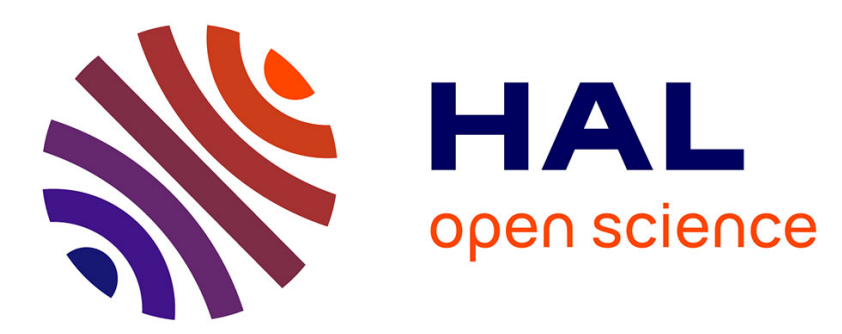

\title{
Mechanisms Governing the Transfer of Pure and Plant Matrix Carotenoids Toward Emulsified Triglycerides
}

Pascale Goupy, Claude Genot, Faiza Hammaz, Charlotte Halimi, Catherine Caris-Veyrat, Patrick Borel

\section{- To cite this version:}

Pascale Goupy, Claude Genot, Faiza Hammaz, Charlotte Halimi, Catherine Caris-Veyrat, et al.. Mechanisms Governing the Transfer of Pure and Plant Matrix Carotenoids Toward Emulsified Triglycerides. Molecular Nutrition and Food Research, 2020, 14 p. 10.1002/mnfr.201900911 . hal-02475159

\section{HAL Id: hal-02475159 \\ https://hal.science/hal-02475159}

Submitted on 20 Apr 2021

HAL is a multi-disciplinary open access archive for the deposit and dissemination of scientific research documents, whether they are published or not. The documents may come from teaching and research institutions in France or abroad, or from public or private research centers.
L'archive ouverte pluridisciplinaire HAL, est destinée au dépôt et à la diffusion de documents scientifiques de niveau recherche, publiés ou non, émanant des établissements d'enseignement et de recherche français ou étrangers, des laboratoires publics ou privés. 


\section{CAROTENOIDS TOWARD EMULSIFIED TRIGLYCERIDES.}

3

4

Pascale GOUPY ${ }^{1}$, Claude GENOT $^{2}$, Faiza HAMMAZ ${ }^{3}$, Charlotte HALIMI ${ }^{3}$, Catherine CARIS-VEYRAT ${ }^{1}$, Patrick BOREL $^{3 *}$

${ }^{1}$ UMR408 SQPOV « Sécurité et Qualité des Produits d'Origine Végétale », INRA, Avignon Université, F-84000 Avignon, France.

${ }^{2}$ UR1268 BIA « Biopolymères Interactions Assemblages », INRA, F-44316 Nantes, France.

${ }^{3}$ C2VN, INRAE, INSERM, Aix Marseille Univ, F-13005, Marseille, France.

Keywords: $\beta$-carotene, lutein, lycopene, phospholipids, serum albumin.

*Corresponding author:

Patrick.Borel@univ-amu.fr

UMR INRAE/INSERM/AMU "Center for CardioVascular and Nutrition Research of Marseille"

\section{Faculté de Médecine}

27, boulevard Jean Moulin

13005 Marseille, France

Phone: +33 (0)4 91324277

Abbreviations: $\beta$-carotene ( $\beta C$ ), lycopene (LYC), lutein (LUT), phospholipids (PL), bovine serum albumin (BSA), triglycerides (TG). 


\section{Abstract}

Scope: to assess the role of factors assumed to be involved in the transfer of carotenoids from plant matrices to dietary emulsions in the upper digestive tract. Methods: We first measured transfer as a function of time of pure $\beta$-carotene $(\beta C)$, lutein (LUT) and lycopene (LYC) to triglyceride (TG) droplets dispersed in water. Then we measured the transfer to TG droplets stabilized with either bovine serum albumin (BSA), phospholipids (PL), or both. Finally, we measured transfer of tomato and spinach puree carotenoids to these emulsions. Main results: the maximal transfer efficiency of the pure carotenoids to uncoated emulsions was very efficient, ranging from 59 to $77 \%$. However, it was dramatically impaired, ranging from 0.5 to $31 \%(\mathrm{p}<0.05)$, when emulsions were stabilized by the emulsifiers. Conversely, when LUT, and to a less extent $\beta \mathrm{C}$, but not LYC, was provided by the vegetable purees, its maximal transfer efficiency was significantly higher for the coated emulsions than for the uncoated one. Conclusions: Emulsifiers can dramatically impair the transfer of pure carotenoids to emulsion TG while they can facilitate the transfer of carotenoids from plant matrices. This suggests that specific interactions between plant matrix compounds and emulsifiers can enhance the transfer efficiency of carotenoids.

(1)

3

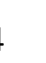

5

46

47




\section{1) Introduction}

\section{(1)}

Carotenoids are hydrophobic plant pigments that belong to the lipid family. Although all their biological effects on the human body are far from being elucidated, some effects are acknowledged, e.g. the provitamin A activity of carotenoids that possess at least one $\beta$-ionone ring in their chemical formulae, and other are more and more assumed, e.g. anti-inflammatory properties ${ }^{[1-4]}$ and role of lutein (LUT) and zeaxanthin in visual function. ${ }^{[5-7]}$

Carotenoid bioavailability is low and modulated by numerous factors. ${ }^{[8-10]}$ It is assumed that this is mostly because they are both very hydrophobic and embedded in plant cells. It is also assumed that carotenoids can only migrate out of plant cells when these cells are broken, either through food processing, cooking or chewing. Indeed, carotenoids are deposited in different forms within plant cells, e.g. chloroplasts, chromoplasts, lipid-dissolved forms, ${ }^{[11]}$ and it has been suggested that cells walls and chromoplast substructure represent important barriers for their bioaccessibility. ${ }^{[12,13]}$ Thus, carotenoids hardly integrate in mixed micelles, which are involved in their transport to their site of absorption. ${ }^{[14,15]}$ In fact, although during digestion it has been shown that a fraction of carotenoids directly transfers from plant matrices into micelles ${ }^{[16]}$ because of their high hydrophobicity most of the carotenoids present in plant food matrices transfer first to the triglyceride (TG) phase of the food bolus, ${ }^{[17]}$ which is more or less dispersed as a so-called 'dietary emulsion'. ${ }^{\text {[1, }}$ 19] Furthermore, because of their high hydrophobicity, non-oxygenated carotenoids, i.e. carotenes, are mostly solubilized in the core of the emulsion lipid droplets, i.e. in the TG phase, ${ }^{[20,21]}$ while oxygenated carotenoids, i.e. xanthophylls, are solubilized both in the core of the lipid droplets and in the phospholipid monolayer located at the droplet surface. ${ }^{[22]}$ Indeed, dietary lipid droplets are coated by surfaceactive molecules, namely emulsifiers, naturally present in foods or secreted in the intestinal lumen during digestion. ${ }^{[23,24]}$ The main dietary emulsifiers are phospholipids (PL) and the 
proteins that possess hydrophilic and hydrophobic sequence(s) of amino acids that allow them to adsorb at and stabilize the lipid droplet interface. ${ }^{[25]}$

Although the transfer of carotenoids from plant matrices to dietary emulsions has been suggested decade ago, and although several studies have studied the transfer of carotenoid to oil in vitro, ${ }^{[12,26-30]}$ in vivo experiments dedicated to measure the carotenoid transfer from plant matrices to dietary emulsions are scarce. In fact, to our knowledge there is only one clinical study dedicated to this topic. ${ }^{[31]}$ In that study it has been shown that the three main dietary carotenoids, i.e. $\beta$-Carotene $(\beta C)$, lycopene (LYC) and LUT, can be transferred from plant matrices to dietary emulsions in the human stomach during digestion. More precisely, up to about $60 \%$ carrot puree $\beta$-Carotene $(\beta C)$ was transferred in the fat phase of the meal after $3 \mathrm{~h}$ digestion. A second interesting observation of this study was that the transfer efficiency of carotenoids can be very different depending on carotenoid species and on the nature of the food matrix. For instance, the maximal transfer efficiency of tomato puree LYC was less than $10 \%$ while that of carrot puree $\beta C$ was about $60 \%$.

Yet, factors that are involved in these very different transfer efficiencies have not been completely understood so far and it is for example not known whether the differences between the maximal transfer efficiency of LYC and $\beta C$ were due to the different physicochemical properties of the carotenoids, as observed for the transfer of carotenoids between TG and mixed micelles ${ }^{[32]}$ or to the effect of the food matrix, ${ }^{[9,11,33-36]}$ which can result both from physical barriers or from interactions with molecules that are associated with carotenoids in plant matrices. ${ }^{[11]}$ Furthermore, concerning the dietary emulsions, the effects of emulsifier(s) that coat the lipid droplets, and which can also be present as isolated or as molecular assemblies in the aqueous medium, on the transfer efficiency of carotenoids has never been investigated, although a recent study has suggested a role of PL on the transfer efficiency of tomato LYC to oil. ${ }^{[29]}$ Yet, we hypothesize that these compounds might impair the transfer of carotenoids to oil 
droplets by creating a barrier hindering the direct diffusion of carotenoid to this hydrophobic phase. ${ }^{[37]}$

This project aimed at assessing the role of the three main factors that we assume to modulate the transfer of carotenoids to dietary emulsions, i.e. carotenoid species, presence and species of emulsifiers, and presence and nature of the plant matrix. To that aim, we first compared the transfer of three pure solid carotenoids, with contrasted physicochemical characteristics, ${ }^{[10,32]}$ to uncoated sunflower oil droplets dispersed in a slightly acid aqueous medium ( $\mathrm{pH} 4.0$ ), considered as representative of the physicochemical conditions found in the first step of digestion, that is during bolus formation in the stomach. Then, we studied the effect of the presence and the type of emulsifiers that coat these droplets by measuring the transfer of the pure carotenoids to the TG droplets stabilized with either a model protein: bovine serum albumin (BSA), well-known for its emulsifying properties, a mixture of PL, which was mainly composed of L- $\alpha$-phosphatidylcholine, or a mixture of the two emulsifiers, which best mimic what happens in vivo. We finally assessed the role of the environment of carotenoids in plant matrix by measuring the transfer of carotenoids from tomato and spinach purees to either the uncoated TG droplets or to the TG droplets coated with the emulsifiers. The results obtained have allowed us to suggest fundamental mechanisms that govern this first step of carotenoid bioavailability. 


\section{2) Materials and methods}

119

120

121

122

123

124

125

126

127

\section{Chemicals and vegetable sources of carotenoids}

Acetone, dichloromethane, methanol, $n$-hexane, ethyl-acetate and 2-propanol were purchased from Fisher-Scientific (Illkirch, France). Sodium acetate, sodium chloride and formic acid were purchased from VWR (Fontenay-sous-Bois, France). Ammonium formate, (all-E)- $\beta$ carotene $(\beta C)(\geq 95 \%)$, bovine serum albumin (BSA) (fraction V A-9647, MW ca. $66500 \mathrm{~g}$ $\mathrm{mol}^{-1}$ ), and trans- $\beta$-Apo- $8^{\prime}$-carotenal (> 95\%) were purchased from Sigma-Aldrich (St Quentin-Fallavier, France). L- $\alpha$-phosphatidylcholine from dried egg yolk ( $>=50 \%$ TLC) was from Sigma-Aldrich (Buchs, Switzerland). Certificate of analysis of the lot used in this study indicated that it contained $76 \%$ L- $\alpha$-phosphatidylcholine. A previous analysis of another lot of the same product by our lab (data not shown) showed that this product also contained some triglycerides (TG) and a mixture of phosphatidylethanolamine, sphingomyelin, lysophosphatidylcholine and phosphatidylinositol. It is therefore called the "PL mixture" thereafter in the manuscript. (all-E)-Lycopene (LYC) $(>90 \%$ ) was purified in the lab as previously described ${ }^{[38]}$ from a tomato oleoresin provided by Conesa (Badajoz, Spain). (all-E)Lutein (LUT) (>99 \%) was also purified in the lab, ${ }^{[39]}$ but from a Naturex (Montfavet, France) oleoresin. Sunflower oil (Lesieur, France) was bought in a local supermarket. We chose this food oil because it is a commonly consumed oil. However, we acknowledge that other results could have been obtained with other oils. Indeed, it has been shown that the solubility of carotenoids increased when the chain-length of the triglycerides' fatty acids decreased. ${ }^{[22]}$

Two common dietary sources of carotenoids were used in this study: tomato, which is rich in LYC and $\beta C$ and also contains LUT, and spinach, which is rich in LUT and also contains $\beta$ C. Cold break tomato puree (cultivar Terradou ${ }^{\circledR}$ ) was prepared according to previous work ${ }^{[40]}$. We chose this source of tomato because it was very well characterized and it allowed 
us to compare data obtained in this study with data obtained with the same tomato matrix in a previous study by our lab on the diffusivity of lycopene from tomato puree to oil. ${ }^{[29]}$ Canned spinach leaves were bought in a local supermarket (Casino brand). We chose canned spinach leaves in order to compare data obtained in this study with data obtained with the same spinach matrix in a previous study by our lab on the effect of spinach matrix on lutein bioaccessibility and bioavailability. ${ }^{[33]}$ To have a homogeneous matrix, spinach leaves were diluted four times with deionized water, i.e. $100 \mathrm{~g}$ drained spinach leaves were dispersed in a total volume of 400 $\mathrm{mL}$ water, and transformed in puree using a rotor stator homogenizer Silentcrusher (Heidolph, Grosseron, France) set at $16000 \mathrm{rpm}$ for $2 \mathrm{~min}$ under dim light at room temperature.

\section{Phospholipid purification}

In order to measure the association of carotenoids with pure PL we eliminated residual neutral lipids (mainly triglycerides) from the egg yolk product rich in L- $\alpha$-phosphatidylcholine according to the method modified from Gladkowski et al. ${ }^{[41]}$ In summary, absolute ethanol (10 $\mathrm{mL}$ ) was added to $2 \mathrm{~g}$ of the egg yolk product in a $50 \mathrm{ml}$ flask placed on a magnetic stirrer. After solubilization, the solution was placed at $-20^{\circ} \mathrm{C}$ for $4 \mathrm{~h}$ and then centrifuged at $3000 \mathrm{x} \mathrm{g}$ at $4^{\circ} \mathrm{C}$ for $5 \mathrm{~min}$. The supernatant was recovered, placed again at $-20^{\circ} \mathrm{C}$ for $12 \mathrm{~h}$ and centrifuged at $3000 \mathrm{xg}$ at $4{ }^{\circ} \mathrm{C}$ for $5 \mathrm{~min}$. The new supernatant was recovered and evaporated under vacuum. Cold acetone $(20 \mathrm{~mL})$ was finally added to solubilize contaminating TG and the mixture was shake in ice. PL, which are insoluble in acetone, were precipitated, collected and washed with cold acetone to remove remaining TG still present. Purified PL were stored at $-20^{\circ} \mathrm{C}$ until used. 
Standard solutions of carotenoids

Standard solutions of carotenoids $\left(\sim 50 \mu\right.$ mol. $\left.\mathrm{L}^{-1}\right)$ were prepared by dissolving the dry standards in dichloromethane. Apo-8'-carotenal was dissolved in absolute ethanol. The integrity of the carotenoid standards and their concentrations in the stock solutions were checked and calculated by using a Specord S-600 diode-array UV-vis spectrophotometer (Analytical Jena, Saint-Aubin, France). The following molar absorption coefficients were used: for LYC in dichloromethane, $140516 \mathrm{~L} \cdot \mathrm{mol}^{-1} \cdot \mathrm{cm}^{-1}$ at $444 \mathrm{~nm}$ for LUT in methanol containing $1 \%$ dichloromethane, $108900 \mathrm{~L} \cdot \mathrm{mol}^{-1} . \mathrm{cm}^{-1}$ at $457 \mathrm{~nm}$ for apo-8'-carotenal in absolute ethanol.

\section{CAR extraction from the vegetable sources of carotenoids}

Tomato and spinach puree carotenoids were extracted using a method described by

Serino et al. ${ }^{[42]}$ with few modifications. More precisely, $500 \mathrm{mg}$ of spinach puree or $250 \mathrm{mg}$ of tomato puree were put in $2 \mathrm{ml}$ Eppendorf tubes containing around $100 \mathrm{mg}$ of $0.1 \mathrm{~mm}$ Zirconia/ Silica Beads (Biospec products, Bartlesville, USA) and $50 \mu \mathrm{L}$ of a stock solution of apo-8'carotenal $(33 \mu \mathrm{M}$ in ethanol) used as internal standard. Then, $100 \mu \mathrm{L}$ of saturated aqueous $\mathrm{NaCl}$ solution, $100 \mu \mathrm{L}$ of $\mathrm{n}$-hexane, $200 \mu \mathrm{L}$ of dichloromethane and $800 \mu \mathrm{L}$ of ethyl-acetate were successively added, each time followed by a 60 s full-speed high-energy shaking, using a was re-extracted with the same successive solvents and the second upper phase was pooled with the first one and evaporated under argon to obtain the extracted lipids containing the carotenoids. Carotenoids were quantified by HPLC as described in the dedicated paragraph. 
$0.05 \pm 0.01 \mathrm{mg} / 100 \mathrm{~g}$ LUT. Spinach puree contained $2.34 \pm 0.02 \mathrm{mg} / 100 \mathrm{~g}$ fresh weight LUT and $0.36 \pm 0.02 \mathrm{mg} / 100 \mathrm{~g} \beta \mathrm{C}$.

\section{$\underline{\text { Preparation of sunflower oil-in-water emulsions }}$}

Four different emulsions were prepared: sunflower oil droplets dispersed in an aqueous medium (uncoated emulsion), oil droplets stabilized by BSA (BSA-emulsion), oil droplets stabilized by PL (PL-emulsion) or oil droplets stabilized by both emulsifiers (BSA/PLemulsion). We studied the effect of the combination of the two emulsifiers because in most meals the two types of emulsifiers are simultaneously present and partition at the droplet interface according to their own interfacial properties. All systems were adjusted to $\mathrm{pH} 4.0$ because we aimed to study the transfer of carotenoids from vegetable food matrices to dietary emulsions that occurs in the human stomach, ${ }^{[31]}$ and $\mathrm{pH} 4.0$ is the mean $\mathrm{pH}$ observed in the human stomach after ingestion of vegetable-rich meals that contained tomato and spinach purees. ${ }^{[31]}$ The proportions of the different component families, i.e. TG, PL and proteins, were representative of those occurring in the human gastrointestinal tract during digestion of a typical western meal. ${ }^{[31]}$ Sunflower oil was chosen as the source of long chain TG because it is one of the most consumed one in Western countries. For the preparation of the uncoatedemulsions, $4 \mathrm{~g}$ of sunflower oil were added to $32 \mathrm{~mL} 10 \mathrm{mM}$ acetate buffer at $\mathrm{pH} 4.0$ in a 60 $\mathrm{mL}$ bottle. Then, the mixture was vigorously agitated by using a magnetic barrel stirrer for 1 min at room temperature. Obviously, this mixture was unstable, and the TG droplets coalesced very quickly. Thus, it was used extemporaneously in the transfer experiments described in the dedicated paragraph. The PL, BSA and BSA/PL-emulsions were prepared as follows: the emulsifiers were first dispersed in $32 \mathrm{~mL} 10 \mathrm{mM} \mathrm{pH} 4.0$ acetate buffer (BSA: $4.44 \mathrm{~g} / \mathrm{L}$; PL-rich 
mixture: $3.13 \mathrm{~g} / \mathrm{L}$; BSA $4.44 \mathrm{~g} / \mathrm{L}+$ PL-rich mixture $3.13 \mathrm{~g} / \mathrm{L}$ ). Then the emulsifiers were dispersed using a rotor stator homogenizer (Heidolph Silent Crusher M) at 24,000 rpm for 2 min at room temperature. Then, $4 \mathrm{~g}$ of sunflower oil was added to the emulsifier dispersions. The mixtures were then sonicated (Qsonica sonicator and a 3/4" probe) to obtain normal distributions with mean particle sizes ranging between 2 and $4 \mu \mathrm{m}$, which was close to the size of lipid droplets observed in the human gastrointestinal tract during digestion. ${ }^{[19]}$ The sonication conditions were empirically adjusted to obtain emulsions that were stable during the transfer experiments. Concerning the BSA and BSA/PL-emulsions the power was set at 20$25 \mathrm{~W}$, the amplitude at 40 , and the sonication was performed in ice for 4 periods of $30 \mathrm{~s}$ with rest intervals of $30 \mathrm{~s}$. Concerning the PL-emulsions, the power was set at $40 \mathrm{~W}$, the amplitude at 80 , and the sonication was performed in ice for 4 periods of $60 \mathrm{~s}$ with rest intervals of $30 \mathrm{~s}$. The final $\mathrm{pH}$ of the mixtures was adjusted to 4.0 with either $\mathrm{HCl}$ or $\mathrm{NaOH}$ depending on the initial $\mathrm{pH}$.

\section{Measurement of the size distribution of the emulsion lipid droplets}

The size distribution of the stabilized emulsions was measured using a Mastersizer 2000 laser diffraction instrument (Malvern Panalytical SARL, Orsay, France). Each emulsion was prepared 3 times independently as described above and 2 measures were performed on each sample. Thus, means were obtained from 6 values. The parameters modes: D [4,3], D [3,2], Dv10, Dv50 and Dv90 were calculated using the instrument's software. Calculations were performed using the Mie theory with the following refractive indexes: 1.475 for oil, 1.333 for the dispersant, and an absorbance value of 0.01 for the emulsion particles. The specific surface area $\left(\mathrm{m}^{2} / \mathrm{g}\right)$ was calculated by the software (total area of the particles divided by the total weight of oil). This calculation was based on the assumption that the particles were both spherical and non-porous. ${ }^{[43]}$ 
Measurement of the zeta potential of the emulsion lipid droplets and of the tomato and spinach

242

243

244 particles

The zeta potential was measured on stabilized emulsions or vegetable puree particles dispersed and diluted with $10 \mathrm{mM}$ acetate buffer at $\mathrm{pH} 4.0$ (dilution ratio between 50 and 5000). Measurements were performed in triplicate at $25^{\circ} \mathrm{C}$ using the Wallis zeta potential analyser (Cordouan Technologies, Pessac, France). Ten sequential runs were averaged to obtain one value. Zeta potentials were calculated using Henry’s equation ${ }^{[44]}$ and the Smoluchowski approximation. A mean zeta-potential measurement was obtained from three independent samples. Main settled parameters were: laser power $70 \%$, low resolution $(5 \mathrm{~Hz})$, dielectric constant (relative permittivity) 78.06, applied field $21.47 \mathrm{mV}$, solvent (water) viscosity $0.888 \mathrm{cP}$, carrier frequency $8223 \mathrm{~Hz}$.

\section{Determination of the apparent solubility of carotenoids in aqueous solutions containing the} $\underline{\text { emulsifiers }}$

We deposited about $50 \mu \mathrm{g}$ of each pure carotenoid solubilized in dichloromethane in 8 $\mathrm{mL}$ glass tubes that possessed plugs and we dry evaporated them at room temperature under argon and gentle agitation (Vibrax Orbital movement shaker model VXR basic set at $500 \mathrm{rpm}$ ). The gentle agitation was performed to obtain a homogeneous film of carotenoids glued at the bottom of to the glass tube. Then, we added $2 \mathrm{~mL} 0.1 \mathrm{mM} \mathrm{pH} 4.0$ acetate buffer containing either BSA, PL-rich mixture, purified PL, or a 50/50 mixture of BSA and PL-rich mixture, or purified PL. Emulsifier concentrations were the same than those used to make the emulsions, i.e. $4.44 \mathrm{~g} / \mathrm{L}$ for BSA and 3.125 g/L for PL-rich mixture and purified PL. After $4 \mathrm{~h}$ incubation at $37^{\circ} \mathrm{C}$ under gentle agitation (same shaker and setting than above), the solutions were centrifugated $\left(1000 \mathrm{x}\right.$ g for $5 \mathrm{~min}$ at $\left.4{ }^{\circ} \mathrm{C}\right)$ to discard potential carotenoid crystals remaining in 
suspension. Carotenoids remaining in the aqueous medium were theoretically associated with the emulsifiers because of their very low solubility in water, were quantified by UPLC.

\section{Measurement of the transfer of carotenoids from either pure carotenoids or vegetable puree}

\section{carotenoids to either uncoated or coated emulsions}

To study the transfer of pure carotenoids to the TG phase of the different emulsions we dry evaporated about $50 \mu \mathrm{g}$ of each carotenoid in $8 \mathrm{~mL}$ glass tubes as described above. Then, we added $2 \mathrm{~mL}$ of the different emulsions. Note that in the case of uncoated emulsions we first added $2 \mathrm{~mL} 10 \mathrm{mM}$ pH 4.0 acetate buffer then we added $0.25 \mathrm{~mL}$ sunflower oil in order to avoid direct contact of the oil with the carotenoid film. We then closed the tubes with their plugs, and we incubated them at $37^{\circ} \mathrm{C}$ under constant stirring for 240 min (same shaker and setting than above). After $0.5,15,30,60,90,120,150$ and $240 \mathrm{~min}, 1.5 \mathrm{~mL}$ of the mixtures were sampled (we used one glass tube per incubation time), and TG, which was theoretically rid of emulsifiers, ${ }^{[20,21]}$ was recovered as described below.

To study the transfer of vegetable puree carotenoids to the TG phase of the different emulsions we first diluted the vegetable purees with ultrapure $\mathrm{H}_{2} \mathrm{O}$ (MilliQ system) to obtain liquid mixtures that could be readily blended with the emulsions and that provided a quantity of carotenoids nearly equivalent to that of pure carotenoids. Indeed, these dilutions provided around 20 and $50 \mu \mathrm{g}$ of total carotenoids per incubation condition for spinach and tomato purees, respectively. Two $\mathrm{mL}$ of the emulsions were then added, and the mixtures were incubated at $37^{\circ} \mathrm{C}$ under constant stirring (same apparatus and speed than above) for 240 min. Samples of the mixtures were collected at the same interval times than above. The TG phase, free of emulsifiers, was recovered as described below.

To break the emulsions and recover the TG phase free of emulsifiers and of vegetable puree particles, samples that came from the transfer experiments were centrifuged for 15 min at 
$5,000 \times \mathrm{g}$, frozen overnight at $-20^{\circ} \mathrm{C}$, thawed at room temperature and centrifuged again for 30 $\min$ at $5,000 \mathrm{x}$. This allowed to obtain a floating layer of pure $\mathrm{TG}$ at the top of the tubes. Furthermore, to avoid any contamination of the TG samples with emulsifiers that are located at the interface between the TG and the aqueous phase, ${ }^{[20,21]}$ only a fraction of the TG layer floating at the top of the centrifuge tube, about $0.1 \mathrm{~g}$, was collected. Each experiment was performed in triplicate.

\section{CAR quantification by UPLC}

Carotenoids extracted from the vegetable puree matrices or incorporated in the emulsion TG phases recovered after the transfer experiments were analyzed by HPLC as described thereafter. The dried extracts of carotenoids obtained after tomato and spinach puree extraction were first dissolved in $1 \mathrm{~mL}$ of dichloromethane/methanol (50/50; v/v) that contained $3.3 \mu \mathrm{M}$ apo-8'-carotenal as internal standard. The recovered emulsion TG phases containing the transferred carotenoids were weighed and dissolved in $200 \mu \mathrm{L}$ of 2-propanol and $800 \mu \mathrm{L}$ of dichloromethane/methanol (50/50; v/v), which also contained $3.3 \mu \mathrm{M}$ apo-8'-carotenal as internal standard. The analyses were performed using an ACQUITY UPLC® system (Waters Corp., Milford, USA) linked to a diode array detector 190-800 nm. Empower ${ }^{\mathrm{TM}} 2$ software (Waters Corp.) was used for instrument control and chromatogram integrations. The separation was carried out using an ACQUITY UPLC® HSS T3 C18 column $(150 \mathrm{~mm} \times 2.1 \mathrm{~mm}$, internal diameter $1.8 \mu \mathrm{m}$; Waters Corp.), which temperature was set at $50{ }^{\circ} \mathrm{C}$. Solvents used were methanol/water (80:20, v/v) (solvent A) and ethyl acetate (solvent B) containing $0.2 \%$ formic acid. Gradient conditions were: A, 100\%, 0-2 min; A, 100-30\%, 2-20 min; A, 30-20\%, 20-22 min; A, 20-100\%, 22-23 min; and returned to the initial conditions (solvent A, 100\%) for a 4 min re-equilibration period ${ }^{[45]}$. The system operating pressure was 800 bars at initial gradient conditions and injection volume was $7.5 \mu \mathrm{L}$ with partial-loop with needle overfill injection. 
The peaks were detected at 440 and $470 \mathrm{~nm}$ for LUT and the carotenes (LYC and $\beta C$ ), respectively. The limit of quantification was $2 \mathrm{ng}$ for $\mathrm{LYC}, 3 \mathrm{ng}$ for $\beta \mathrm{C}$ and $0.2 \mathrm{ng}$ for LUT.

\section{Calculations and statistics}

The transfer efficiency was defined as the percentage of carotenoids recovered in the TG phase of the emulsions (measured mg CAR/g TG x $0.2 \mathrm{~g} \mathrm{TG}$, which was the total amount of TG provided by the $2 \mathrm{~mL}$ emulsion used in each experiment) relative to the amount of carotenoids, either pure or provided by the vegetable purees, that was incubated with the emulsions. The maximal transfer efficiency was the highest measured transfer efficiency that was mostly obtained after $240 \mathrm{~min}$. incubation. using ANOVA followed by post hoc Tukey-Kramer tests. All $p$ values under 0.05 were considered significant. Statistical comparisons were performed using StatView software, version 5.0 (SAS Institute Inc., Cary, NC).

\section{Dosage information / Dosage regimen}

The doses of pure carotenoids used in this study were non supraphysiological/pharmacological. Indeed, we incubated $50 \mu \mathrm{g}$ pure carotenoids per tube while the average intake of each of these phytochemicals is higher than $1 \mathrm{mg} /$ person/d. ${ }^{[46]}$ 


\section{3) Results}

\section{Main characteristics of the different emulsions}

Because we hypothesized that differences in transfer efficiency, and/or transfer rate, of carotenoids between the different emulsions could be due to different characteristics of the emulsions we measured both their lipid droplet size distribution and their electric charge. Indeed, for a given amount of oil, the lower the size of the droplets the higher the emulsion specific surface area $\left(\mathrm{m}^{2} / \mathrm{g}\right.$ oil $)$ and thus the surface of the lipid droplets that can get in contact with the carotenoids. Note that the characteristics of the uncoated emulsion were not measured because, as stated in the material and method section, it was composed of coarse nonhomogeneous TG droplets, apparently in the $\mathrm{mm}$ range, which coalesced very quickly. As expected, all coated emulsions exhibited monomodal particle size distributions with droplet sizes in the range 0.5-4 $\mu \mathrm{m}$ (supporting information Fig. S4). Several parameters characterizing the lipid droplet size distributions of the different coated emulsions are shown in table 1. All of them, except the D3,2, were significantly $(\mathrm{p}<0.05)$ different between the 3 emulsions. The specific surface area of the PL-emulsion was about $60 \%$ higher than that of the BSA-emulsion and about two times higher than that of the BSA/PL-emulsion. We calculated, from the mass charge of BSA at the interface (theoretical surface load) given by the literature and the emulsion's specific surface area, that in the BSA-emulsion about $25 \%$ of the BSA was adsorbed at the oil-water interface, meaning that around $75 \%$ of the BSA remained in the aqueous phase. ${ }^{[4]}$ Concerning the PL-emulsion, $65 \%$ of PC were adsorbed at the interface.

355 Finally, concerning the BSA/PL-emulsion, about $30 \%$ PL present in the system were enough to cover the TG droplets and we assumed that all BSA remained in the aqueous phase. Indeed,

357 it is acknowledged that there is a preferential adsorption of low-molecular weight emulsifiers as compared to high molecular weight emulsifiers such as proteins. ${ }^{[47-49]}$ 

possible to measure it for the uncoated emulsion because this emulsion was too unstable. The zeta potential of the BSA-emulsion was $24.6 \pm 0.4 \mathrm{mV}$, that of the PL-emulsion was $-9.3 \pm 0.4$ $\mathrm{mV}$, while that of the BSA/PL-emulsion was intermediate, i.e. $8.3 \pm 0.6 \mathrm{mV}$. These zeta potentials were significantly different $(\mathrm{p}<0.05)$. Concerning the vegetable puree particles, they had close negative charges, i.e. $-11.5 \pm 0.2$ and $-10.2 \pm 0.2 \mathrm{mV}$ for the tomato and spinach puree particles, respectively.

\section{Stability of carotenoids in the emulsions}

To accurately measure the transfer efficiency of carotenoids toward the TG phase of the studied emulsions, and because it has been shown that Z-isomerization of LYC can enhance the transfer efficiency, ${ }^{[30,50]}$ we checked whether carotenoids were stable in these emulsions during the experiment. To that purpose we quantified carotenoid concentrations as well as possible formation of carotenoid (Z)- isomers, at regular time intervals from up to $240 \mathrm{~min}$, in the TG phase of BSA/PL-emulsions (data not shown). Neither the total amount of each carotenoid incorporated in TG nor their $(Z)$ to (all-E)-isomer ratios significantly changed ( $p>0.05$ ) over time, i.e. all along the $240 \mathrm{~min}$. This shows that the studied carotenoids were relatively stable in the studied emulsions, at least in the conditions of our experiments, i.e. $240 \mathrm{~min}$ at $\mathrm{pH} 4.0$ at $37^{\circ} \mathrm{C}$, which agrees with recent results showing that $\beta \mathrm{C}$ is not significantly degraded in the upper human gastrointestinal tract lumen during digestion. ${ }^{[51]}$

\section{Apparent solubility of carotenoids in emulsifiers dispersed in aqueous medium}

Results reported in table 2 show that the apparent solubility of carotenoids in the emulsifiers dispersed in an aqueous medium depended both on carotenoid and emulsifier species. For example, while about $11 \%$ of LUT was recovered in PL-rich mixture dispersion, 
only about $0.5 \%$ of $\beta \mathrm{C}$ was. Concerning LYC it was not detected in the aqueous medium whatever the emulsifier(s) used. PL were better than BSA to solubilize $\beta C, 0.52$ vs $0.03 \%$ $(\mathrm{p}<0.05)$, while it was the opposite for LUT, 19.54 vs $11.12 \%(\mathrm{p}<0.05)$. The apparent solubility of both carotenoids were higher when BSA and PL were mixed than when they were used alone $(p<0.05)$. Finally, when purified PL were used instead of the PL mixture, which was a mixture of PL, lysophosphatidylcholine, TG and sphingomyelin (see material and methods), the apparent solubility of $\beta \mathrm{C}$ was non significantly different, 0.77 vs $0.52 \%$, while that of LUT was significantly decreased, 5.82 vs $11.12 \%$. Also, the mixture of BSA plus purified PL led to a lower apparent solubility of LUT than the mixture of BSA plus PL mixture.

\section{Transfer of carotenoids toward uncoated sunflower oil droplets}

Figure 1 shows the time-dependence of carotenoid incorporation (wt \% of initial amount of each carotenoid added in the system) in uncoated sunflower oil dispersed in acetate buffer at $\mathrm{pH}$ 4.0. As shown in Fig. 1A the transfer of pure solid carotenoids, which initially glued to the wall of the glass tubes, to the uncoated TG droplets, increased as a function of time to nearly reach a plateau. The maximal transfer efficiency was about $59 \%$ for LUT and about 72 and $77 \%$ for LYC and $\beta$, respectively (Fig. 5). When carotenoids were provided in the tomato puree matrix the curves of transfer showed very different shapes (Fig. 1B) and the maximal transfer efficiencies of all the carotenoids were significantly $(p<0.05)$ lower than that of the pure carotenoids (supporting information Fig. S1A, S2A and S3A). The maximal transfer efficiency for LYC was about $12 \%$ and that for $\beta C$ and LUT were about 27 and $28 \%$, respectively (supporting information Fig. S3A, S2A and S1A). Finally, when carotenoids were in spinach puree (Fig. 1C) the maximal transfer efficiencies of carotenoids to uncoated TG droplets were the lowest with values of about $8 \%$ for LUT and about $5 \%$ for $\beta \mathrm{C}$ (supporting 
information Fig. S1A and S2A). Note that there is no data on the transfer of LYC from spinach puree because this carotenoid is not significantly present in this vegetable.

\section{Transfer of carotenoids toward sunflower oil droplets emulsified with BSA}

The only difference between Fig. 2 and Fig. 1 is that the carotenoids were incubated with TG droplets stabilized with BSA instead of uncoated TG droplets. Fig. 2A shows that the presence of BSA dramatically impaired the transfer of pure carotenoids to TG located in the core of these droplets. Indeed, the maximal transfer efficiencies of the solid pure carotenoids ranged between 0.5 and $4.4 \%$ as compared to values higher than $59 \%$ for pure solid carotenoids to the uncoated emulsion (Fig. 5). In contrast, when the carotenoids were provided by tomato puree (Fig. 2B), their maximal transfer efficiencies for the BSA-emulsion (7.6\% for LYC, $26.8 \%$ for $\beta \mathrm{C}$, and $51.6 \%$ for LUT; Fig. $6 \mathrm{~B}$ ) were higher than those observed when they were provided as pure solids ( $0.5 \%$ for LYC, $0.7 \%$ for $\beta C$, and $4.4 \%$ for LUT; Fig. 5). The same observation could be made for the maximal transfer efficiencies of carotenoids provided in spinach puree. They were higher for the BSA-emulsion then for the uncoated emulsion.

\section{Transfer of carotenoids toward sunflower oil droplets emulsified with PL}

Fig. 3A and 5 show that the maximal transfer efficiencies of the pure carotenoids to these emulsions ranged between $1.5 \%$ for LYC to $30.9 \%$ for LUT. When the carotenoids were provided by tomato puree (Fig. 3B), the maximal transfer efficiency dramatically increased for LUT, reaching $72.5 \%$ (Fig 6A). The maximal transfer efficiency of LYC also increased, from 1.5 to $8.5 \%$ (Fig. 5C and 6C). Finally, when the carotenoids were provided by the spinach puree (Fig. 3C), their maximal transfer efficiencies were about $41 \%$ and $10 \%$ for LUT and $\beta C$ (Fig. 7A and 7B), respectively. 
Transfer of carotenoids toward sunflower oil droplets stabilized with both BSA and PL

Fig. 4A shows that the maximal transfer efficiency of pure carotenoids to TG located in BSA/PL-emulsions was very low. Indeed, it ranged between $0.9 \%$ for LYC to $8 \%$ for $\beta \mathrm{C}$ (Fig. 5C, A and B). As observed for the two other emulsions, when LUT was provided in tomato puree its maximal transfer efficiency to the TG of the BSA/PL-emulsion dramatically increased $(\mathrm{p}<0.05)$ reaching about $81 \%$ (Fig. 6A) as compared to pure LUT (7.0\%, Fig. 5A). The maximal transfer efficiencies of the two other carotenoids also significantly $(\mathrm{p}<0.05)$ increased reaching about 10 and $32 \%$ for tomato puree $\mathrm{LYC}$ and $\beta \mathrm{C}$ (Fig. $6 \mathrm{C}$ and $\mathrm{B}$ ) as compared to pure carotenoids, 0.9 and $8.0 \%$, respectively (Fig. 5C and B). The curves of transfer of spinach carotenoids to the BSA/PL-emulsion are shown in Fig. 4C and the corresponding maximal transfer efficiencies are shown in Fig. 7. In summary, the spinach- $\beta C$ maximal transfer efficiency was about $14 \%$ and that of spinach-LUT was about $50 \%$.

\section{Comparison of the maximal transfer efficiencies of pure carotenoids toward sunflower oil when} this oil was either uncoated or emulsified with PL, BSA or both

Fig. 5 to 7 give a summary of the maximal transfer efficiencies measured in the transfer experiments shown in Fig. 1 to 4 . They allow us to compare the maximal transfer efficiency measured for each CAR, either pure or incorporated in the two different vegetable purees, with each different species of emulsion.

Concerning the transfer of pure carotenoids, two general observations can be made. First, the transfer was dramatically impaired when the TG were coated with the emulsifiers (Fig. 5). Second, the lowest maximal transfer efficiencies were obtained when BSA was used as the only emulsifier. Regarding the effect of carotenoid species, it is noteworthy that LUT had the lowest maximal transfer efficiency to uncoated TG, i.e. about 59\%, while LYC and $\beta C$ exhibited close maximal transfer efficiencies for this emulsion, i.e. about 72 and $77 \%$ 
respectively. This ranking markedly changed in the presence of emulsifiers with LYC having the lowest maximal transfer efficiency, regardless the emulsifiers.

Comparison of the maximal transfer efficiencies of tomato puree carotenoids toward sunflower oil when this oil was either uncoated or emulsified with PL, BSA or both

The maximal transfer efficiencies of tomato puree LUT to the different emulsion species were always higher than those of the two other tomato carotenoids (Fig. 6). The maximal transfer efficiencies of the three tomato carotenoids were always higher when PL and BSA were both present, i.e.in the BSA/PL-emulsions, than when they were used alone.

Comparison of the maximal transfer efficiencies of spinach puree carotenoids toward sunflower oil when this oil was either uncoated or emulsified with PL, BSA or both

The first observation that arises from Fig. 7 is that, conversely to what was observed with pure carotenoids (Fig. 5), the lowest maximal transfer efficiencies of both spinach carotenoids were observed with the uncoated emulsion. Moreover, the effect of the emulsifiers on the maximal transfer efficiencies of the spinach carotenoids depended on carotenoid species. Indeed, concerning spinach LUT, PL led to a significantly $(\mathrm{p}<0.05)$ higher maximal transfer efficiency than BSA while it was the opposite for $\beta C$.

\section{Comparison of the maximal transfer efficiency of LUT toward the different emulsions when}

\section{LUT was provided either as a pure solid or incorporated in tomato or spinach puree}

Results presented in supporting information Fig. S1 are not commented because they are presented, in another way, in Fig. 5 to 7. However, we believe that showing these results this way can help the reader to better see another interesting result of this study, that is the effect of the food matrix on the transfer of LUT to the different emulsion species. 
484 Comparison of the maximal transfer efficiency of $\beta C$ toward the different emulsions when $\beta C$ 485 was provided either as a pure solid or incorporated in tomato or spinach puree

486 Same comment for supporting information Fig. S2 than for supporting information Fig.

487 S1, but concerning $\beta C$.

488

489 Comparison of the maximal transfer efficiency of LYC toward the different emulsions when $490 \quad$ LYC was provided either as a pure solid or incorporated in tomato puree

491 Same comment for supporting information Fig. S3 than for supporting information Fig.

492 S1 and S2 but concerning LYC. Obviously, there is no data for spinach LYC because this 493 carotenoid is not present in this vegetable. 


\section{4) Discussion}

Before discussing the results in details it should be mentioned that the transfer values that we observed for the vegetable puree carotenoids to the BSA/PL-emulsion, which is the emulsion assumed to best mimic the dietary emulsions that exist in vivo, showed similar trends than what was observed in vivo. ${ }^{[31]}$ Indeed, we measured about $10 \%$ maximal transfer efficiency for tomato LYC that can be compared to about $6 \%$ recovered in the fat phase of a meal observed in vivo after $3 \mathrm{~h}$ digestion, ${ }^{[31]}$ and about $50 \%$ for spinach LUT as compared to about $30 \%$ measured in vivo. ${ }^{[31]}$

In the first part of this study we aimed to obtain basic data on the transfer efficiency of carotenoids to TG. We thus measured the transfer efficiency of pure carotenoids toward uncoated TG droplets dispersed in an aqueous medium. Results showed that this transfer was very efficient. Indeed, the maximal transfer efficiency ranged between 59 and $77 \%$ depending on carotenoid species. To explain this observation, we hypothesize that the transfer efficiency depends both on the collisions between the agitated TG droplets and the film of carotenoids stuck to the glass tubes, and on the hydrophobic attraction between carotenoids and TG molecules when TG droplets enter in contact with the solid pure carotenoid film. Indeed, the transfer of nonpolar molecules from a polar environment to a nonpolar environment is driven both by the free energy gain ${ }^{[52]}$ and the hydrophobic force. ${ }^{[53]}$ The involvement of the hydrophobic force is further supported by previous finding ${ }^{[28]}$ and by the fact that the maximal transfer efficiency of LUT was lower than that of $\beta C$ and LYC, i.e. 59 vs 77 and $72 \%$ respectively, which can be related to their respective hydrophobicity schematically reflected by their $\log P$, i.e. 11.78 vs 15.51 and 15.19 , respectively (www.chemspider.com). Nevertheless, the use of $\log P$, which measures the partition of a molecule between octanol and water, appears not to be accurate to predict the solubility of carotenoids in oil because (Z)-isomers of 
lycopene, which have the same $\log P$ than the (all-E)-isomer, are more soluble in oil than the (all-E)-isomer. ${ }^{[30,50]}$ Nevertheless, because carotenoid hydrophobicity has also been correlated with transfer of carotenoids from tomato and carrot based matrices to oil, ${ }^{[28]}$ as well as with their transfer efficiency from lipid droplets toward mixed micelles, ${ }^{[32,54]}$ these results suggest that the hydrophobic force is a key player in carotenoid bioaccessibility.

Because dietary fats are coated with amphiphilic dietary molecules in the upper gastrointestinal tract, ${ }^{[18]}$ the second part of this study was dedicated to assessing the effect of the main dietary emulsifiers, i.e. phospholipids and proteins, on the transfer of pure solid carotenoids to the TG located in the core of lipid droplets. ${ }^{[20-22]}$ When TG droplets were emulsified in the presence of the emulsifiers, the maximal transfer efficiencies of pure carotenoids were dramatically impaired (Fig. 5). Furthermore, the ranking of carotenoids regarding their maximal transfer efficiency markedly changed with pure LYC having the lowest maximal transfer efficiency, regardless the emulsifier, meaning that the inhibitory effect of the emulsifiers on the transfer of carotenoids was higher for LYC than for $\beta C$ and LUT. This inhibition was a priori surprising because, as expected, when the TG were stabilized by the emulsifiers the size of the emulsion lipid droplets decreased and the specific surface area, and thus the available surface of exchange between carotenoids and TG, dramatically increased (table 1). Thus, the mechanism(s) that lead(s) to the inhibitory effect of the emulsifiers on the transfer of carotenoids far outweigh(s) the hypothesized potential of the emulsifiers in increasing transfer by increasing the surface of transfer. Several inhibitory mechanisms can be proposed. First, it can be hypothesized that, when the emulsions stabilized with the emulsifiers were incubated with the solid films of pure carotenoids, a fraction of the carotenoids could have been incorporated/associated to the fraction of the emulsifiers that remained dispersed in the aqueous medium, ${ }^{[55,56]}$ becoming not available for the transfer to the lipid droplets. Indeed, the results obtained in this study on the apparent solubility of carotenoids in the emulsifiers 
544 confirm that these compounds can associate with PL ${ }^{[52,57,58]}$ and BSA. ${ }^{[59-61]}$ They further show 545 that the apparent solubility in the emulsifiers is mainly determined by carotenoid species, the carotenoid with two polar groups, LUT, being better associated with the emulsifiers than the two other carotenoids, likely because it associates more easily with amphiphilic molecules. Nevertheless, such a mechanism is not sufficient to explain the inhibitory effect of the emulsifiers on the transfer of carotenoids because the transfer of LUT, which was by far the carotenoid that showed the greatest apparent solubility in PL and BSA (table 2), was less affected by the presence of the emulsifiers than LYC, which was not significantly associated with the emulsifiers (Fig. 5). We thus hypothesize that the emulsifiers adsorbed at the droplet interface could also create a physical barrier (or an energy barrier) that impairs the contact of carotenoids with TG and thus impairs their transfer into the lipid droplets. Furthermore, we hypothesize that this barrier is most important for the carotenoids that are not soluble in the emulsifiers than for those who are. Indeed, we assume that carotenoids solubilized in the emulsifier located at the droplet interface ${ }^{[22]}$, and thus in contact with the TG core of the droplets, can easily transfer to the TG phase, while carotenoids not solubilized in the interface emulsifiers can hardly transfer. This hypothesis explains why the transfer of pure LYC, which was not soluble in the emulsifiers, was more impaired by the emulsifiers than that of the two other pure carotenoids (Fig. 5). Thus, when TG are coated with these emulsifiers, the transfer efficiency of pure carotenoids to TG results from opposite effects: negative effects due to both the quenching of the hydrophobic force by the emulsifier barrier between carotenoids and TG and to the trapping of a fraction of carotenoids by the fraction of emulsifiers that remain in the aqueous phase, ${ }^{[22,55,62]}$ and positive effects due to both the facilitated transfer of carotenoids that are incorporated in the fraction of emulsifiers located at the lipid droplet interface, and to the higher surface of exchange induced by the emulsifiers. Obviously, these hypotheses should be verified by dedicated experiments and we cannot extrapolate these observations to the effect 
of other dietary emulsifiers on the transfer of other carotenoid species. Nevertheless, our results and their interpretations allow us to hypothesize that the transfer efficiency of any carotenoid species to the TG phase of an emulsion stabilized with amphiphilic molecules will mainly depend on the ability of these carotenoids to associate with the amphiphilic molecules located at the interface and to those solubilized in the aqueous phase.

The third part of this study aimed at assessing whether plant matrices, which contain physical barriers that can impair carotenoid diffusion, ${ }^{[13,26]}$ and which also contain carotenoids that can be in different physical states and that can be associated with different matrix molecules, ${ }^{[11]}$ can significantly affect the transfer efficiency. To that aim we chose two vegetables that are usual dietary sources of carotenoids, i.e. tomato ${ }^{[63]}$ and spinach, ${ }^{[33]}$ and we turned them into puree to mimic the size of their particles in the stomach during digestion. After having studied the transfer of carotenoids in the same conditions than those used for the pure carotenoids, we first observed that the transfer efficiency of the carotenoids incorporated in these matrices to the uncoated TG droplets was markedly lower than that of the pure carotenoids (supporting information Fig. S1A, S2A and S3A). To explain this observation, we first hypothesize that this is because carotenoids in plant cells have to cross different barriers that impair their release from the cells. ${ }^{[12,13]}$ Nevertheless, this hypothesis disagrees with the observation that the vegetable puree carotenoids were, in most cases, better transferred to the coated emulsions than the pure carotenoids (supporting information Fig. S1 to S3). Therefore, we suggest that some compounds in the vegetable purees are able to improve the transfer by a mechanism that far outweighs the inhibitory effect of the cell barriers. A second hypothesis to explain the lower transfer of vegetable puree carotenoids to uncoated TG droplets, as compared to pure carotenoids, is that plant cells contain amphiphilic molecules, e.g. proteins and PL, ${ }^{[64]}$ that adsorb at the uncoated TG droplet interface and that create a barrier that impairs the transfer. A second, and very intriguing, observation was that the transfer of both spinach and 
tomato puree LUT was significantly improved by the presence of the emulsifiers (supporting information Fig. S1B, S1C and S1D vs supporting information Fig. S1A). This phenomenon was not observed for tomato $\beta \mathrm{C}$ and LYC (supporting information Fig. S2 and supporting information Fig. S3) and it was of less amplitude for spinach $\beta \mathrm{C}$ (supporting information Fig. S2). To explain this phenomenon, we suggest that the complexes of molecules that were associated with LUT in spinach and tomato purees and with $\beta C$ in spinach puree, or which had been associated with these carotenoids after the transformation of these matrices in purees, associate very efficiently to the TG droplets coated with emulsifiers. The electric charges of the coated or uncoated TG droplets and of the vegetable purees (Table 2) cannot explain why the plant complexes of molecules apparently readily associate with coated TG droplets, while they do not to uncoated TG droplets. Thus, we hypothesize that spinach and tomato LUT, as well as spinach $\beta \mathrm{C}$, were associated with molecules that facilitate anchoring of these complexes to interfaces coated with emulsifiers. Furthermore, the fact that the transfer efficiency of tomato LYC was not enhanced by the presence of emulsifiers (supporting information Fig. S3) was perhaps because LYC in tomatoes is present as crystals in chromoplasts ${ }^{[11]}$ and these crystals are not coated by the molecules that, we hypothesized to, have a high affinity for interfaces coated with emulsifiers. A third observation on the transfer efficiency of the vegetable puree carotenoids to emulsion TG was that this transfer was always more efficient for tomato LUT and $\beta C$ than for spinach LUT and $\beta C$ (supporting information Fig. S1 and supporting information Fig. S2). We hypothesis, but this should be verified in a dedicated study, that carotenoids located in these two different plant matrices could have to cross different biological barriers, ${ }^{[12,13]}$ to go out of these matrices. Another possibility is that the size of the cells or the percentage of remaining intact cells was different in the two purees. Whatever the mechanism, the fact that the transfer is different in the two different plant matrices is in agreement with the very variable effect of different plant matrices on carotenoid bioaccessibility. ${ }^{[13,36]}$. 
In summary, this study has allowed us to suggest fundamental mechanisms governing the transfer of carotenoids to the TG phase of dietary emulsions. We have first suggested that this transfer is basically governed by the hydrophobic force between carotenoids and TG. Then we have shown that amphiphilic molecules that coat TG droplets strongly impair the transfer of pure carotenoids, likely by creating a barrier between them and TG. Furthermore, the transfer of carotenoids that are significantly solubilized in the amphiphilic molecules is less affected. We then found that plant carotenoids hardly transfer to uncoated TG droplets, and we have suggested that this is because they have to cross structural barriers but it is also likely that amphiphilic molecules present in plant matrices coat the interface and hinder the transfer. Conversely, we found that spinach and tomato puree LUT, and to a less extent spinach $\beta C$, were better transferred to TG droplets coated with emulsifiers than to uncoated TG droplets and we hypothesized that this is because lipid/protein complexes that are present with these carotenoids in the studied vegetable matrices facilitate anchoring of these complexes to coated lipid droplets. Further experiments are needed to complete this pioneering study. Indeed, it is likely that digestive enzymes that hydrolyze lipids and proteins in the gastric and intestinal lumen, as well as bile salts that adsorb at the lipid droplet interface during digestion, modify the transfer rate and/or the transfer efficiency of carotenoids. It is also likely that different results would have been obtained with other types of food oil. Indeed, carotenoid solubility in TG depends on the type of TG fatty acids. ${ }^{[22]}$ Nevertheless, we believe that a full understanding of the mechanisms that govern the transfer of plant carotenoids to food emulsions will allow researchers to propose new strategies to improve the bioavailability of these phytochemicals. 
641 The authors thank Conesa and Naturex for supplying tomato puree and CAR-rich oleoresins, 642 respectively. Thanks also to Stéphanie Oriol for photographing the lycopene crystals shown in $643 \quad$ Fig 1 to 3.

644

645 Author contributions:

646 The authors' responsibilities were as follows----PB and CC-V: designed the research and have 647 primary responsibility for final manuscript content. PG and FH: conducted research. PB and 648 CG: analysed data and interpreted them. PB: wrote the paper; and all authors: read and 649 approved the final manuscript.

650

651 Conflicts of interest:

652 None of the authors reported a potential conflict of interest.

653

654 Funding:

655 This project received funding from two incentive programs of the French National Institute for 656 Agricultural Research (INRA): AIC Cepia "Factors limiting the bioaccessibility of carotenoids 657 during digestion" and Action Prioritaire AlimH "Bioavailability of micronutrients". 

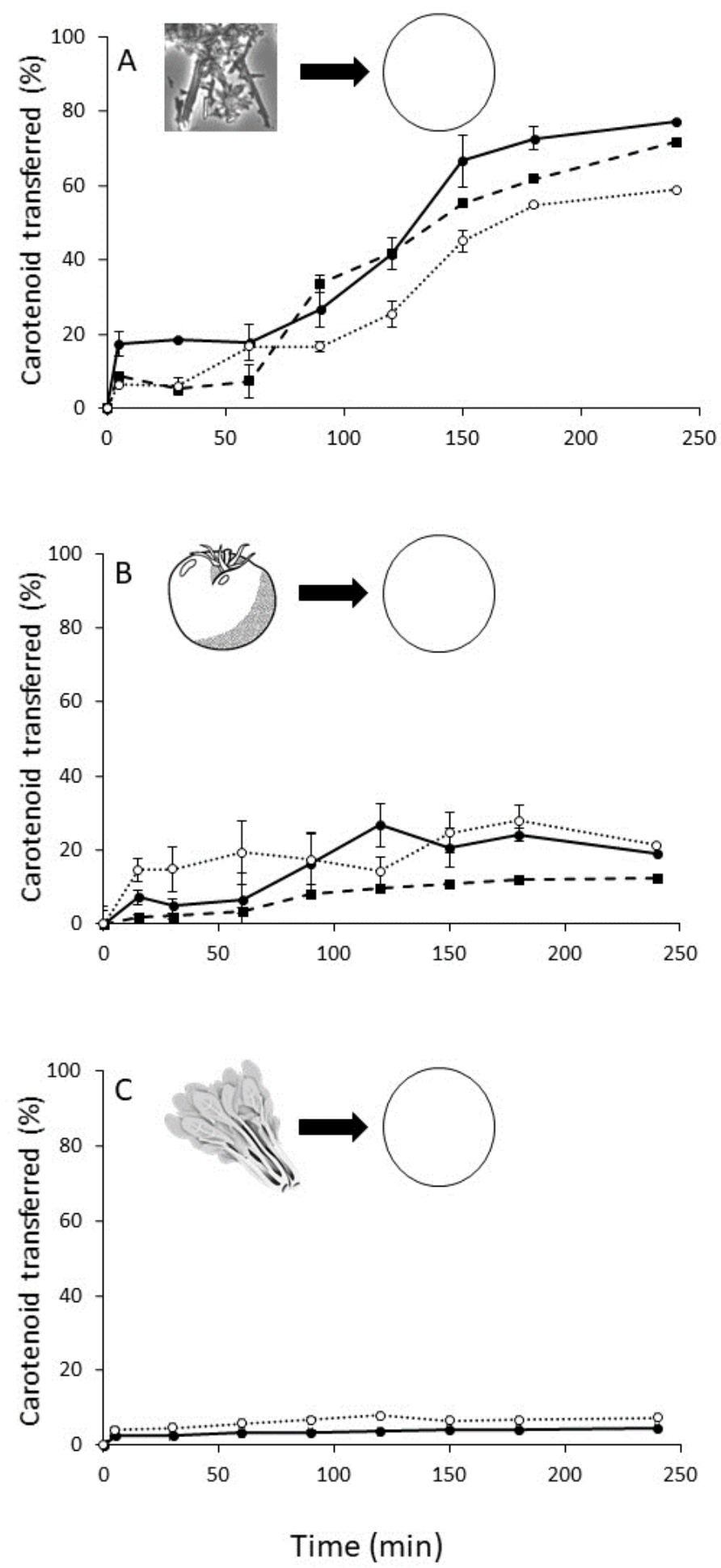

659

660 Figure 1. Transfer of carotenoids to uncoated sunflower TG. 
The graph shows the percent of carotenoid transferred as a function of incubation time (min). A: the photography of lycopene crystals (magnification 100x) and the circle helps to see that this is the transfer of pure solid carotenoids to uncoated TG droplets in aqueous medium. B: the drawing of tomato helps to see that this is the transfer of tomato puree carotenoids, i.e. lycopene (LYC), $\beta$-carotene $(\beta C)$ and lutein (LUT), to uncoated TG droplets. C: the drawing of spinach leaves helps to see that this is the transfer of spinach puree carotenoids, i.e. $\beta$ -
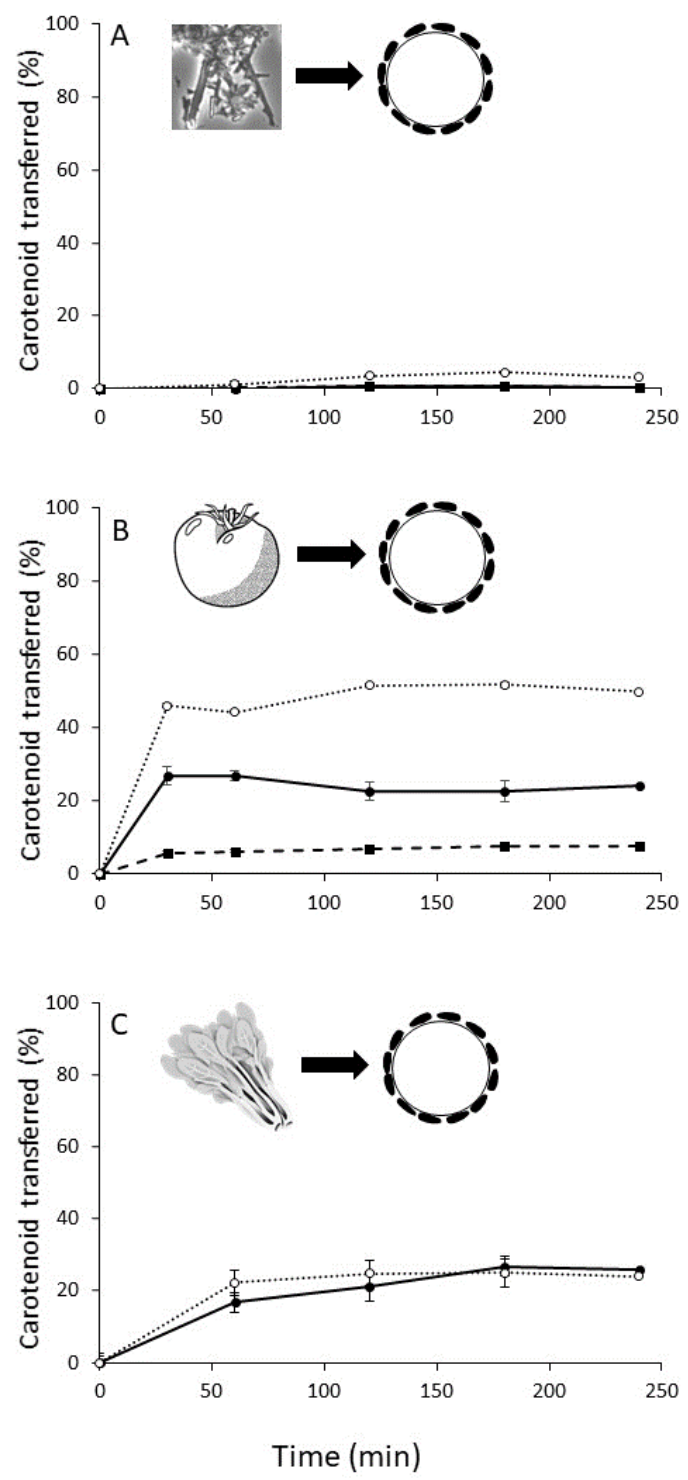
independent experiments.

Figure 2. Transfer of carotenoids to sunflower TG located in emulsions stabilised by BSA. 

of lycopene crystals (magnification 100x) and the circle coated with black forms helps to see that this shows the

672 transfer of pure solid carotenoids to BSA-emulsions. B: the drawing of tomato helps to see that this is the transfer 673 of tomato puree carotenoids, i.e. lycopene (LYC), $\beta$-carotene $(\beta C)$ and lutein (LUT), to BSA-emulsions. C: the 674 drawing of spinach leaves helps to see that this is the transfer of spinach puree carotenoids, i.e. $\beta$-carotene $(\beta C)$
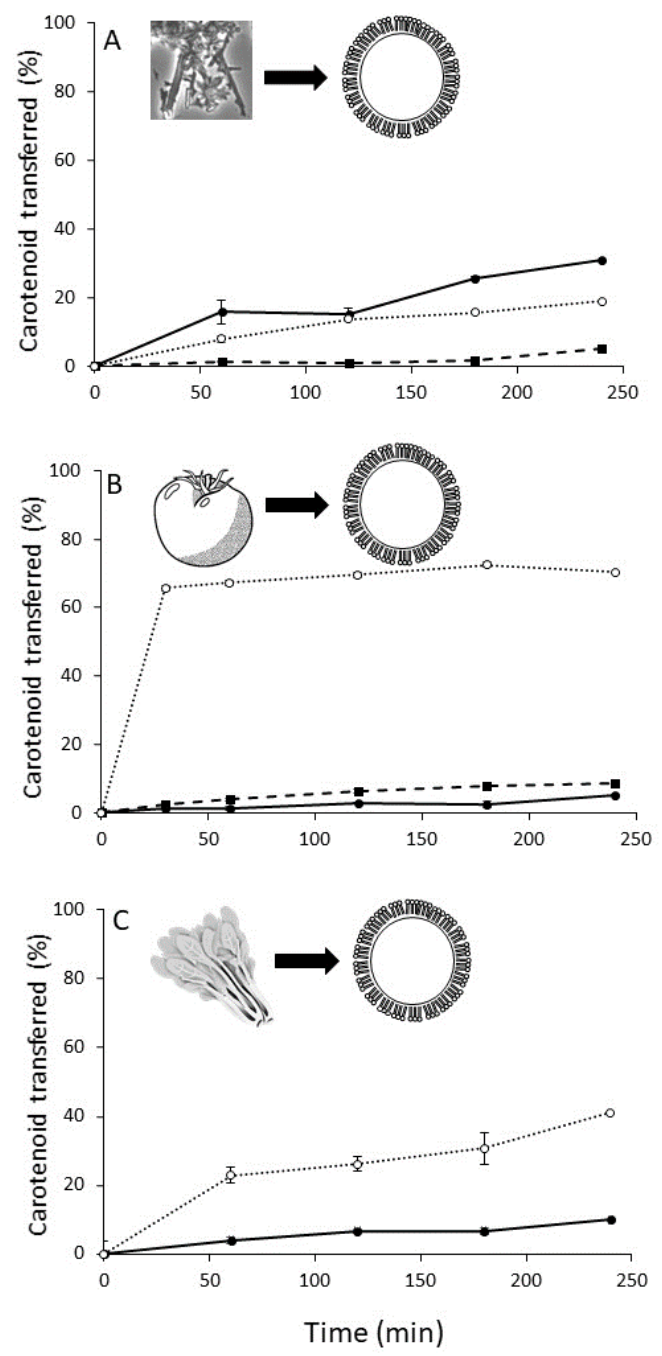

675 and lutein (LUT), to BSA-emulsions. - LYC, • $\beta C$, ○ LUT. Dots represent mean \pm SEM of 4 independent 676 experiments.

677

678 Figure 3. Transfer of carotenoids to sunflower TG located in emulsions stabilised by PL-rich mixture. 
679 The graph shows the percent of carotenoid transferred as a function of incubation time (min). A: the photography of lycopene crystals (magnification 100x) and the circle coated with two arm pins helps to see that this shows the transfer of pure solid carotenoids to PL-emulsions. B: the drawing of tomato helps to see that this is the transfer of tomato puree carotenoids, i.e. lycopene (LYC), $\beta$-carotene $(\beta C)$ and lutein (LUT), to PL-emulsions. C: the drawing of spinach leaves helps to see that this is the transfer of spinach puree carotenoids, i.e. $\beta$-carotene $(\beta C)$
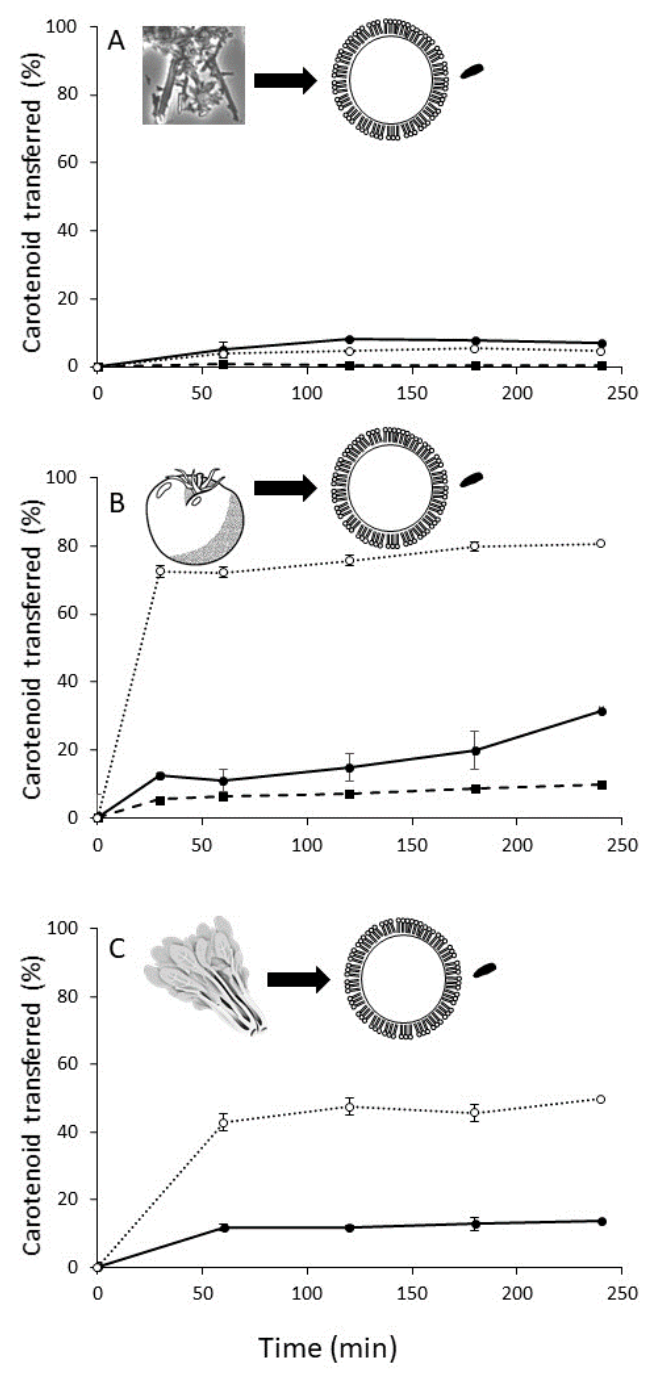
and lutein (LUT), to PL-emulsions. - LYC, - $\beta \mathrm{C}$, ○ LUT. Dots represent mean $\pm \mathrm{SEM}$ of 4 independent experiments.

Figure 4. Transfer of carotenoids to sunflower TG located in emulsions stabilised by a mixture of BSA and PL as emulsifiers. 
689 The graph shows the percent of carotenoid transferred as a function of incubation time (min). A: the photography 690 of lycopene crystals (magnification 100x) and the circle coated with two arm pins, together with a black form 691 close to it, helps to see that this shows the transfer of pure solid carotenoids to BSA/PL-emulsions. B: the drawing 692 of tomato helps to see that this is the transfer of tomato puree carotenoids, i.e. lycopene (LYC), $\beta$-carotene ( $\beta C$ ) 693 and lutein (LUT), to BSA/PL-emulsions. C: the drawing of spinach leaves helps to see that this is the transfer of 694 spinach puree carotenoids, i.e. $\beta$-carotene $(\beta C)$ and lutein (LUT), to BSA/PL-emulsions. — LYC, • $\beta C$, ○ LUT. 695 Dots represent mean \pm SEM of 4 independent experiments.

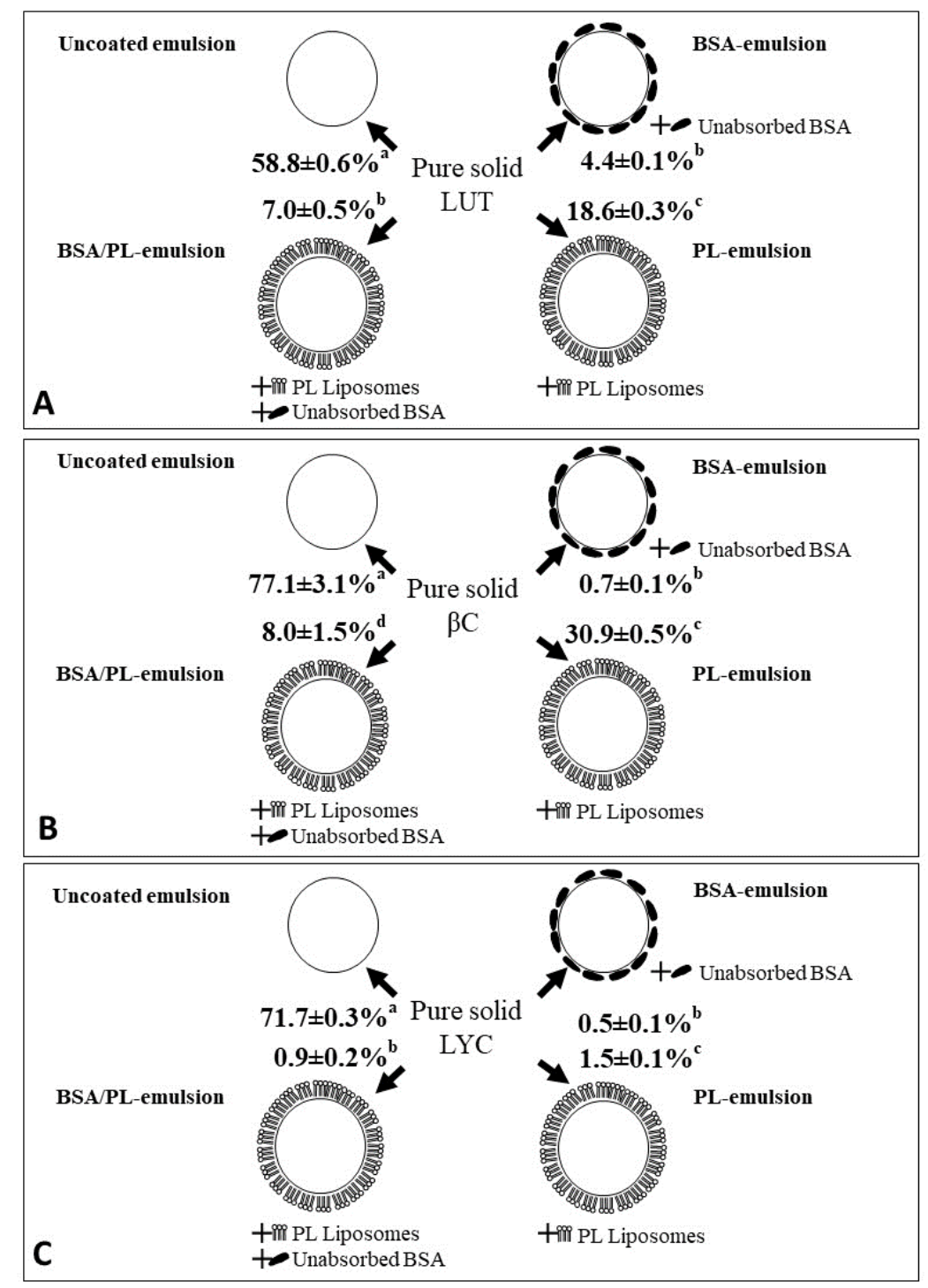


Figure 5. Summary of the maximal transfer efficiencies measured for each pure solid carotenoid in each type of emulsion. The maximal transfer efficiencies were obtained from the curves presented in figures 1 to 4 . A: 698 Maximal transfer efficiency of pure lutein (LUT) to the different emulsions, B: Maximal transfer efficiency of pure $\beta$-carotene ( $\beta C$ ), C: Maximal transfer efficiency of pure lycopene (LYC). The theoretical localisation of the emulsifiers is presented. For example, it has been calculated (see result section) that $65 \%$ of PL were located at the lipid droplet interface and that the remaining fraction was dispersed in the aqueous phase. Values represent mean \pm SEM of 4 independent experiments. In each figure, means that bear different superscript letters are significantly different $(\mathrm{p}<0.05)$, ANOVA followed by Tukey-Kramer post-hoc test.

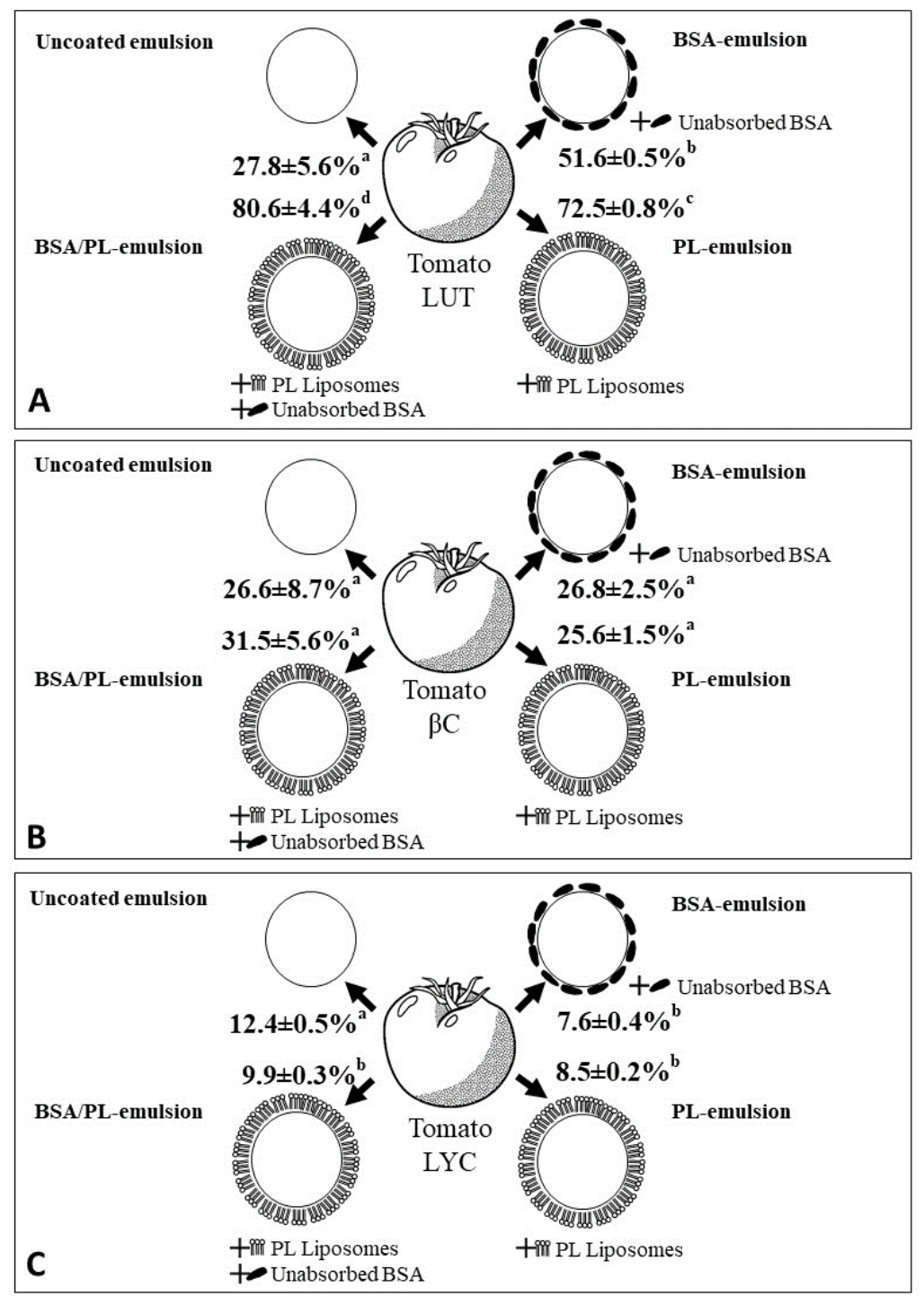


Figure 6. Summary of the maximal transfer efficiencies measured for each tomato puree carotenoid in each type of emulsion. The maximal transfer efficiencies were obtained from the curves presented in figures 1 to 4 . A: Maximal transfer efficiency of pure lutein (LUT) to the different emulsions, B: Maximal transfer efficiency of pure $\beta$-carotene $(\beta C)$, C: Maximal transfer efficiency of pure lycopene (LYC). The theoretical localisation of the emulsifiers is presented. For example, it has been calculated (see result section) that $65 \%$ of PL were located at the lipid droplet interface and that the remaining fraction was dispersed in the aqueous phase. Values represent mean \pm SEM of 4 independent experiments. In each figure, means that bear different superscript letters are significantly different $(\mathrm{p}<0.05)$, ANOVA followed by Tukey-Kramer post-hoc test.
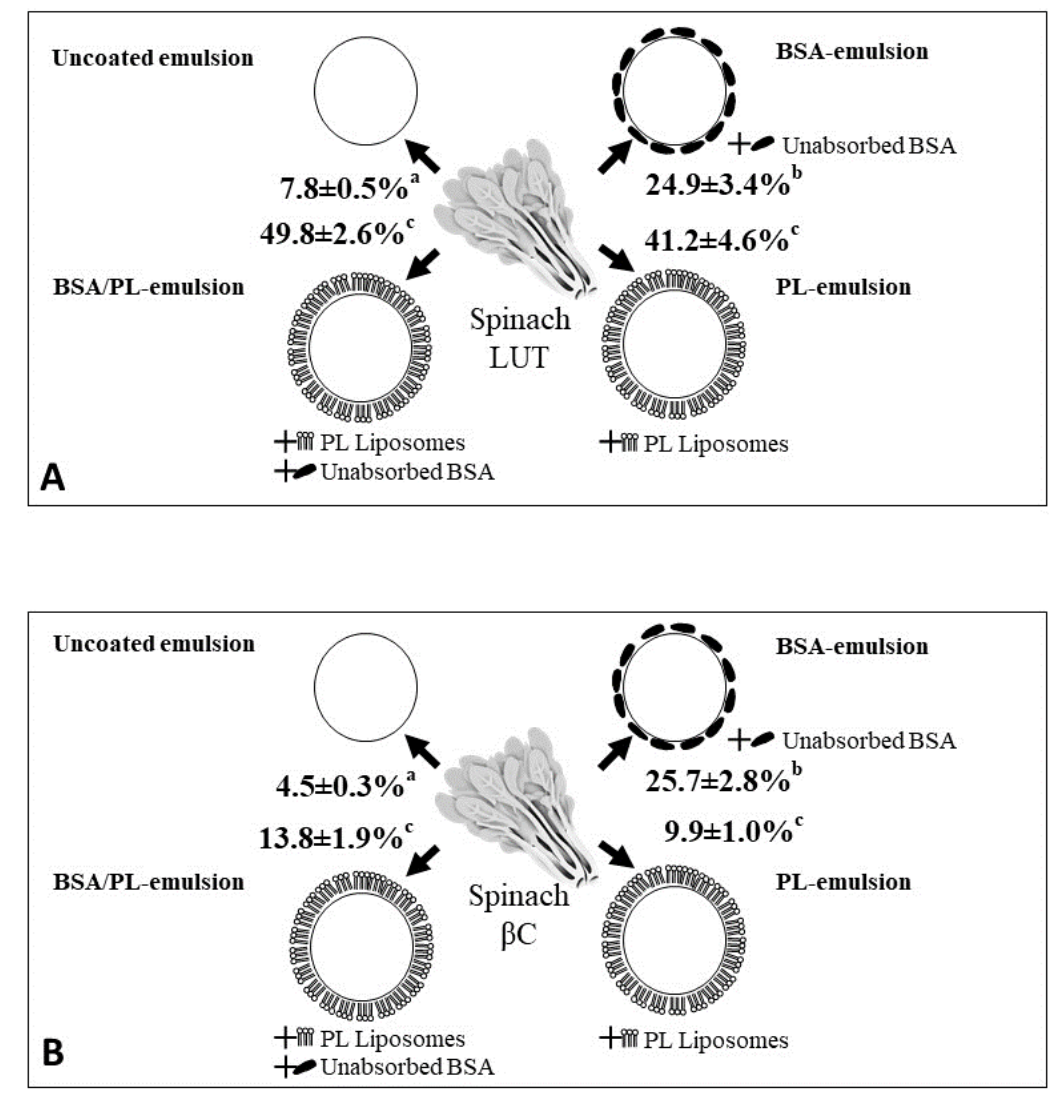
713 Figure 7. Summary of the maximal transfer efficiencies measured for each spinach puree carotenoid in each type

714 of emulsion

715 The maximal transfer efficiencies were obtained from the curves presented in figures 1 to 4. A: Maximal transfer

716 efficiency of pure lutein (LUT) to the different emulsions, B: Maximal transfer efficiency of pure $\beta$-carotene ( $\beta C$ ).

717 The theoretical localisation of the emulsifiers is presented. For example, it has been calculated (see result section)

718 that $65 \%$ of PL were located at the lipid droplet interface and that the remaining fraction was dispersed in the

719 aqueous phase. Values represent mean \pm SEM of 4 independent experiments. In each figure, means that bear different superscript letters are significantly different $(\mathrm{p}<0.05)$, ANOVA followed by Tukey-Kramer post-hoc test. 
Main characteristics of the emulsions stabilised by BSA, PL mixture or both*.

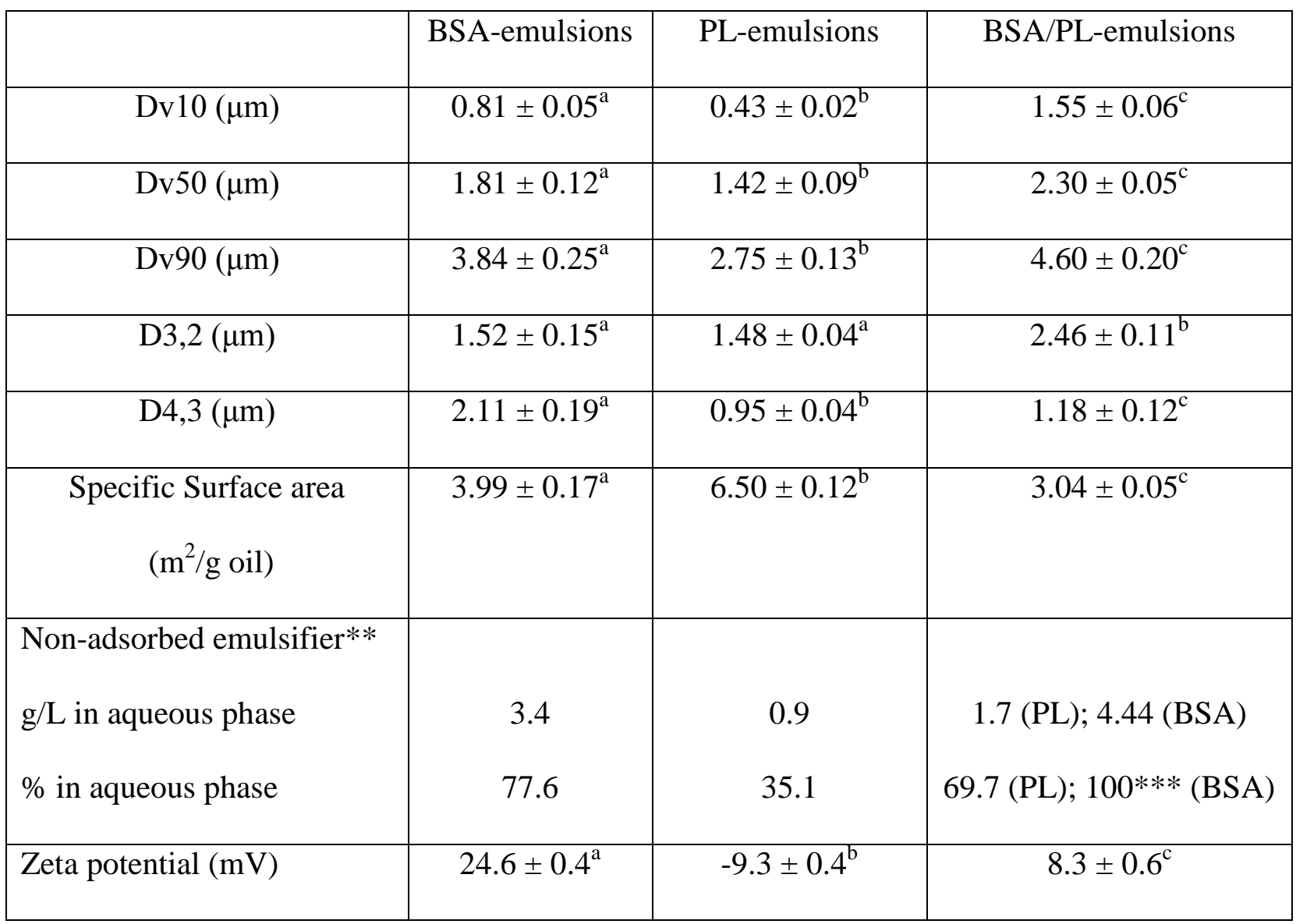

724

Values are means \pm SEM of 6 measurements (3 different emulsions measured 2 times each).

Dv10 is the droplet diameter below which $10 \%$ of the total lipid volume of the sample is contained. Dv50 is the median size. Dv90 is the droplet diameter below which $90 \%$ of the total lipid volume of the sample is contained. D3,2 is the surface mean diameter. D4,3 is the volume mean diameter. In each line, means bearing different letters are significantly different (ANOVA followed by Tukey-Kramer post-hoc test, $\mathrm{p}<0.05)$.

*: it was not possible to measure these characteristics for the uncoated emulsion, i.e. pure TG suspended in aqueous medium, because the TG droplets were very unstable and coalesced very quickly. ${ }^{* *}$ : amounts of emulsifiers not absorbed at the droplet interface have been estimated 
733 using $2 \mathrm{mg} / \mathrm{mL}$ as theoretical surface load for both BSA and PL; ${ }^{[47]} * * *$ : in the presence of PL 734 in excess, BSA is estimated not to be adsorbed at the interface. 
737 Apparent solubility of pure carotenoids in aqueous dispersions of BSA, PL mixture, Purified $738 \quad$ PL or mixtures of the emulsifiers.

\begin{tabular}{|c|c|c|c|c|c|}
\hline & BSA & PL mixture & Purified PL & BSA+PL & BSA+Purified \\
& & & & mixture & PL \\
\hline$\beta C$ & $0.03 \pm 0.02^{\mathrm{a}}$ & $0.52 \pm 0.11^{\mathrm{b}}$ & $0.77 \pm 0.11^{\mathrm{b}}$ & $1.36 \pm 0.21^{\mathrm{c}}$ & $0.65 \pm 0.02^{\mathrm{b}}$ \\
\hline LUT & $19.54 \pm 0.61^{\mathrm{c}}$ & $11.12 \pm 1.46^{\mathrm{b}}$ & $5.82 \pm 3.44^{\mathrm{a}}$ & $43.19 \pm 5.91^{\mathrm{d}}$ & $11.42 \pm 1.53^{\mathrm{b}}$ \\
\hline LYC & ND & ND & ND & ND & ND \\
\hline
\end{tabular}

or a 50/50 mixture of both emulsifiers, and carotenoids recovered in the aqueous dispersions of emulsifiers were quantified by UPLC. *Maximal percentage of carotenoid recovered in dispersions of emulsifiers. Values are mean \pm SEM of 3 to 9 measurements. ND: not detected, i.e. $<0.01 \%$. In each line, means bearing different letters were significantly different (ANOVA followed by Tukey-Kramer post-hoc test, $\mathrm{p}<0.05)$. 


\section{5) References}

746

747

[1] E. Gouranton, C. Thabuis, C. Riollet, C. Malezet-Desmoulins, C. El Yazidi, M. J. Amiot, P. Borel, J. F. Landrier, J Nutr Biochem 2011, 22, 642.

749 [2] A. Kaulmann, T. Bohn, Nutr Res 2014.

750 [3] J. Marcotorchino, B. Romier, E. Gouranton, C. Riollet, B. Gleize, C. Malezet-Desmoulins, J. F. 751 Landrier, Mol. Nutr. Food Res. 2012, 56, 725.

752

[4] S. Fenni, H. Hammou, J. Astier, L. Bonnet, E. Karkeni, C. Couturier, F. Tourniaire, J. F. Landrier, Mol. Nutr. Food Res. 2017, 61, doi: 10.1002/mnfr.201601083.

754 [5] J. Mares, Annu. Rev. Nutr. 2016, 36, 571.

755 [6] P. S. Bernstein, B. Li, P. P. Vachali, A. Gorusupudi, R. Shyam, B. S. Henriksen, J. M. Nolan, Prog. Retin. Eye Res. 2016, 50, 34.

757

[7] E. J. Johnson, Nutr. Rev. 2014, 72, 605.

758

[8] P. Borel, Clin. Chem. Lab. Med. 2003, 41, 979.

759

[9] C. Desmarchelier, P. Borel, Trends Food Sci Technol 2017, 69, 270.

760

[10] T. Bohn, C. Desmarchelier, L. O. Dragsted, C. S. Nielsen, W. Stahl, R. Ruhl, J. Keijer, P. Borel, Mol. Nutr. Food Res. 2017, 61, doi: 10.1002/mnfr.201600685.

762

[11] R. M. Schweiggert, R. Carle, Crit. Rev. Food Sci. Nutr. 2017, 57, 1807.

763

[12] P. Palmero, A. Panozzo, I. Colle, C. Chigwedere, M. Hendrickx, A. Van Loey, Food Chem. 2016, 199, 423.

[13] P. Palmero, L. Lemmens, A. Ribas-Agusti, C. Sosa, K. Met, J. D. Umutoni, M. Hendrickx, A. Van Loey, Food Chem. 2013, 141, 2036.

767 [14] E. Reboul, P. Borel, Prog. Lipid Res. 2011, 50, 388.

768 [15] P. Borel, G. Lietz, A. Goncalves, F. Szabo de Edelenyi, S. Lecompte, P. Curtis, L. Goumidi, M. J. Caslake, E. A. Miles, C. Packard, P. C. Calder, J. C. Mathers, A. M. Minihane, F. Tourniaire, E. Kesse- 
Guyot, P. Galan, S. Hercberg, C. Breidenassel, M. Gonzalez Gross, M. Moussa, A. Meirhaeghe, E. Reboul, J. Nutr. 2013, 143, 448.

[16] G. T. Rich, R. M. Faulks, M. S. Wickham, A. Fillery-Travis, Lipids 2003, 38, 947.

773

[17] G. T. Rich, A. Fillery-Travis, M. L. Parker, Lipids 1998, 33, 985.

774

[18] M. Armand, P. Borel, B. Pasquier, C. Dubois, M. Senft, M. Andre, J. Peyrot, J. Salducci, D. Lairon, 775 Am. J. Physiol. 1996, 271, G172.

776

777

778

779

780

781

782

783

784

785

786

787

788

789

790

791

792

793

794

[19] M. Armand, P. Borel, C. Dubois, M. Senft, J. Peyrot, J. Salducci, H. Lafont, D. Lairon, Am. J. Physiol. $1994,266, \mathrm{G} 372$.

[20] K. W. Miller, D. M. Small, J. Biol. Chem. 1983, 258, 13772.

[21] K. W. Miller, D. M. Small, J. Colloid Interface Sci. 1982, 89, 466.

[22] P. Borel, P. Grolier, M. Armand, A. Partier, H. Lafont, D. Lairon, V. Azais-Braesco, J. Lipid Res. 1996, $37,250$.

[23] M. C. Carey, D. M. Small, C. M. Bliss, Annu. Rev. Physiol. 1983, 45, 651.

[24] B. K. Nordskog, C. T. Phan, D. F. Nutting, P. Tso, Adv Drug Deliv Rev 2001, 50, 21.

[25] C. Genot, T. H. Kabri, A. Meynier, in Food enrichment with omega-3 fatty acids, (Eds: C. Jacobsen, N. S. Nielsen, A. Frisenfeldt Horn, A. D. Moltke Sorensen), Woodhead Publishing Ltd, Cambridge, UK 2013, Ch. 5, 150.

[26] P. Palmero, I. Colle, L. Lemmens, A. Panozzo, T. T. M. Nguyen, M. Hendrickx, A. Van Loey, J. Sci. Food Agric. 2016, 96, 254.

[27] P. Palmero, A. Panozzo, D. Simatupang, M. Hendrickx, A. Van Loey, Food Research International $2014,64,831$.

[28] L. Mutsokoti, A. Panozzo, E. T. Musabe, A. Van Loey, M. Hendrickx, J Funct Foods 2015, 19, 775.

[29] D. Page, C. Labadie, P. Reling, R. Bott, C. Garcia, C. Gaillard, B. Fourmaux, N. Bernoud-Hubac, P. Goupy, S. George, C. Caris-Veyrat, Food Chem. 2019, 274, 500.

[30] J. H. Yu, B. Gleize, L. F. Zhang, C. Caris-Veyrat, C. M. G. C. Renard, Lwt-Food Sci Techno/ 2019, 113. 
[31] V. Tyssandier, E. Reboul, J. F. Dumas, C. Bouteloup-Demange, M. Armand, J. Marcand, M. Sallas, P. Borel, Am J Physiol Gastrointest Liver Physiol 2003, 284, G913.

[32] C. Sy, B. Gleize, O. Dangles, J. F. Landrier, C. C. Veyrat, P. Borel, Mol. Nutr. Food Res. 2012, 56, 1385.

[33] M. Margier, C. Buffiere, P. Goupy, D. Remond, C. Halimi, C. Caris-Veyrat, P. Borel, E. Reboul, Mol. Nutr. Food Res. 2018, 62, e1800185.

[34] T. Bohn, G. J. McDougall, A. Alegria, M. Alminger, E. Arrigoni, A. M. Aura, C. Brito, A. Cilla, S. N. El, S. Karakaya, M. C. Martinez-Cuesta, C. N. Santos, Mol. Nutr. Food Res. 2015, 59, 1307.

[35] J. J. M. Castenmiller, C. E. West, J. P. H. Linssen, K. H. van het Hof, A. G. J. Voragen, J. Nutr. 1999, 129, 349.

[36] E. Reboul, M. Richelle, E. Perrot, C. Desmoulins-Malezet, V. Pirisi, P. Borel, J. Agric. Food Chem. $2006,54,8749$.

[37] X. Yuan, J. Xiao, X. Liu, D. J. McClements, Y. Cao, H. Xiao, Food Chem. 2019, $278,811$.

[38] C. Caris-Veyrat, A. Schmid, M. Carail, V. Bohm, J. Agric. Food Chem. 2003, 51, 7318.

[39] R. E. Kopec, M. Carail, C. Caris-Veyrat, J. Chromatogr. B Analyt. Technol. Biomed. Life. Sci. 2018, $1102-1103,45$.

[40] D. Page, E. Van Stratum, A. Degrou, C. M. Renard, Food Chem. 2012, 135, 2462.

[41] W. Gladkowski, G. Kielbowicz, A. Chojnacka, B. Pisarski, C. Wawrzenczyk, Przem. Chem. 2010, 89, 352.

[42] S. Serino, L. Gomez, G. Costagliola, H. Gautier, J. Agric. Food Chem. 2009, 57, 8753.

[43] P. Chylek, Appl. Opt. 1986, 25, 3166.

[44] A. V. Delgado, F. Gonzalez-Caballero, R. J. Hunter, L. K. Koopal, J. Lyklema, P. International Union of, P. Applied Chemistry, I. T. R. Biophysical Chemistry Division, J. Colloid Interface Sci. 2007, 309, 194.

[45] P. Goupy, M. A. Viana, F. Chemat, C. Caris-Veyrat, Industrial Crops and Products 2013, 44, 496.

[46] R. Estevez-Santiago, B. Beltran-de-Miguel, B. Olmedilla-Alonso, Int. J. Food Sci. Nutr. 2016, 67, 305. 
[47] C. Berton-Carabin, M. H. Ropers, C. Genot, Comprehensive Reviews in Food Science and Food Safety 2014, 13, 945.

[48] J. L. Courthaudon, E. Dickinson, D. G. Dalgleish, J. Colloid Interface Sci. 1991, 145, 390.

[49] A. R. Mackie, A. P. Gunning, P. J. Wilde, V. J. Morris, Langmuir 2000, 16, 2242.

[50] Q. R. Sun, C. Yang, J. Li, H. Raza, L. F. Zhang, J. Food Sci. 2016, 81, C2381.

[51] R. E. Kopec, C. Caris-Veyrat, M. Nowicki, B. Gleize, M. Carail, P. Borel, Am. J. Clin. Nutr. 2018, 108, 803.

[52] K. Makuch, M. Markiewicz, M. Pasenkiewicz-Gierula, Computational and structural biotechnology journal 2019, 17, 516.

[53] M. Azadi, A. V. Nguyen, G. E. Yakubov, Langmuir 2015, 31, 1941.

[54] V. Tyssandier, B. Lyan, P. Borel, Biochim. Biophys. Acta 2001, 1533, 285.

[55] W. A. F. Wan Mohamad, D. McNaughton, M. A. Augustin, R. Buckow, Food Chem. 2018, 257, 361.

[56] W. A. F. Wan Mohamad, R. Buckow, M. Augustin, D. McNaughton, Food Chem. 2017, 233, 197.

[57] W. Grudzinski, L. Nierzwicki, R. Welc, E. Reszczynska, R. Luchowski, J. Czub, W. I. Gruszecki, Scientific reports 2017, 7, 9619.

[58] M. van de Ven, M. Kattenberg, G. van Ginkel, Y. K. Levine, Biophys. J. 1984, 45, 1203.

[59] E. Gouranton, C. E. Yazidi, N. Cardinault, M. J. Amiot, P. Borel, J. F. Landrier, Food Chem. Toxicol. $2008,46,3832$.

[60] B. Rodriguez Galdon, C. Pinto Corraliza, J. J. Cestero Carrillo, P. Macias Laso, Luminescence 2013, 28,765 .

[61] H. T. Chang, H. Cheng, R. M. Han, J. P. Zhang, L. H. Skibsted, J. Agric. Food Chem. 2016, 64, 5951.

[62] A. Mora-Gutierrez, R. Attaie, M. T. Nunez de Gonzalez, Y. Jung, S. Woldesenbet, S. A. Marquez, J. Dairy Sci. 2018, 101, 18.

[63] V. Tyssandier, C. Feillet-Coudray, C. Caris-Veyrat, J. C. Guilland, C. Coudray, S. Bureau, M. Reich, M. J. Amiot-Carlin, C. Bouteloup-Demange, Y. Boirie, P. Borel, J. Am. Coll. Nutr. 2004, 23, 148.

[64] M. Vishnevetsky, M. Ovadis, A. Vainstein, Trends Plant Sci. 1999, 4, 232. 
